\title{
Investigations of Positron Annihilation Radiation
}

\author{
P.A. Milne ${ }^{1}$, J.D. Kurfess ${ }^{2}$, R.L. Kinzer ${ }^{2}$, M.D. Leising ${ }^{3}$ and \\ D.D. Dixon ${ }^{4}$ \\ ${ }^{1}$ NRC/NRL Resident Research Associate, Naval Research Lab, \\ Code 7650, Washington DC 20375 \\ ${ }^{2}$ Naval Research Lab, Code 7650, Washington DC 20375 \\ ${ }^{3}$ Clemson University, Clemson, SC 29631 \\ ${ }^{4}$ Institute of Geophysics and Planetary Physics, Univ. of Cal. Riverside, CA 92521
}

\begin{abstract}
By combining OSSE, SMM and TGRS observations of the galactic center region, Purcell et al. (1997) and Cheng et al. (1997) produced the first maps of galactic positron annihilation. That data-set has been augmented with additional data, both recent and archival, and re-analyzed to improve the spectral fitting. ${ }^{1}$ The improved spectral fitting has enabled the first maps of positronium continuum emission and the most extensive maps of $511 \mathrm{keV}$ line emission. Bulge and disk combinations have been compared with the $511 \mathrm{keV}$ line data, demonstrating that extended bulges are favored over a GC point source for every disk model tested. This result is independent of whether OSSE-only, OSSE/SMM, or OSSE/SMM/TGRS data-sets are used. The estimated bulge to disk ratio (and to a lesser extent the total flux) is shown to be dependent upon the assumption of bulge shape. A positive latitude enhancement is shown to have an effect upon the $\mathrm{B} / \mathrm{D}$ ratio, but this effect is secondary to the choice of bulge shape.
\end{abstract}

\section{INTRODUCTION}

One of the primary objectives of the OSSE instrument on NASA's COMPTON Observatory has been to understand the nature of galactic positron annihilation radiation. Through $8 \frac{1}{2}$ years of observations, $511 \mathrm{keV}$ line emission has been detected, but the emission has never been unambiguously attributable to a given discrete source. The galactic center (GC) region's $511 \mathrm{keV}$ emission was monitored by the Gamma-Ray Spectrometer on-board the Solar Maximum Mission (SMM) (1980-1988), the Transient Gamma-Ray Spectrometer (TGRS) on-board the WIND mission (1995-1997) and with multiple OSSE observations (1991-present). None of these detections require variable sources in addition to the two component models discussed here to explain the measured fluxes (Share et al. 1990, Harris et al. 1998,

1) This paper represents the combination of presentations D-5 and D-12. 
Purcell et al. 1997). These results have supported the suggestion that the majority of the emission is diffuse. Reported here are preliminary results of the extension of the OSSE analysis into three new areas: the inclusion of observations in regions with no a priori expectations of positron annihilation radiation, the extension of the analyzed region to include a larger fraction of the Galaxy, and the mapping of the positronium continuum component (PCONT) of the total annihilation emission. The derived PCONT flux values have a stronger dependence upon fitting the underlying continuum than do the $511 \mathrm{keV}$ line flux values, and thus detailed analyses (model-fitting \& 1D cuts) are only performed upon the $511 \mathrm{keV}$ data-set in this preliminary presentation. ${ }^{2}$

Previously published OSSE results have focussed upon the $511 \mathrm{keV}$ line emission emanating from the central radian of the inner Galaxy, mapping emission from $|l| \leq 33^{\circ},|b| \leq 17^{\circ}$, and model-fitting on a $|l| \leq 90^{\circ},|b| \leq 45^{\circ}, 1^{\circ} \times 1^{\circ}$ grid. In a result first reported at the Fourth Compton Symposium and based on $6+$ years of OSSE data, Purcell et al. 1997 (hereafter PURC97), showed evidence for three components to the $511 \mathrm{keV}$ line emission, (1) an intense slightly extended emission centered in the direction of the GC, (2) a fainter planar emission, and (3) an unexpected enhancement of emission from positive latitudes (PLE). To generate that data-set, two basic types of data were used; (1) standard \& offset pointing data where the scan angle crossed the GC and/or was perpendicular to the galactic plane, and (2) mapping data which searches for emission by observing a large sky region at regularly spaced intervals along a scan path. ${ }^{3}$ The live-time from mapping observations is spread over many more pointings (16 or 32 for mapping versus 39 for standard), so the sensitivity per source pointing achieved is inferior to the standard or offset observations.

The present work relaxes all selection criteria, initially including all observations whose source and background pointings are within the $|l| \leq 90^{\circ},|b| \leq 45^{\circ}$ region. To extract the positronium component from the total emission, three spectral models have been fit to each spectrum (from $60 \mathrm{keV}$ to $700 \mathrm{keV}$ ); (1) a single powerlaw $+511 \mathrm{keV}$ line + PCONT (as fit by PURC97, though they fit from $50 \mathrm{keV}$ $-4 \mathrm{MeV}),(2)$ a power-law with an exponential fall-off $+511 \mathrm{keV}+\mathrm{PCONT}$, (3) a thermal bremsstrahlung model $+511 \mathrm{keV}+\mathrm{PCONT}$. If the best-fitting model (of the three) is deemed to provide an acceptable fit, then that result is selected for subsequent mapping analysis. This has been done for the 1153 observations in the data-set. Fewer than 20 observations have been rejected in this preliminary, allinclusive analysis. The combination of including archival data and the use of new data collected in the $2+$ years since the PURC97 paper has increased the total GC exposure from $1.9 \times 10^{7} \mathrm{det} \cdot \mathrm{s}$ to $8.6 \times 10^{7} \mathrm{det} \cdot \mathrm{s}$, as seen in Figure 1 . As a result of this exposure, the fraction of the GC region $\left(|l| \leq 90^{\circ},|b| \leq 45^{\circ}\right)$ mapped above our exposure threshold has increased from $22 \%$ to $83 \%$.

\footnotetext{
2) Among the potentially important effects not yet addressed are emission from the diffuse cosmicray continuum and a correction for scan-angle dependent background.

3) See PURC97 for details of the OSSE pointing strategies and background techniques.
} 


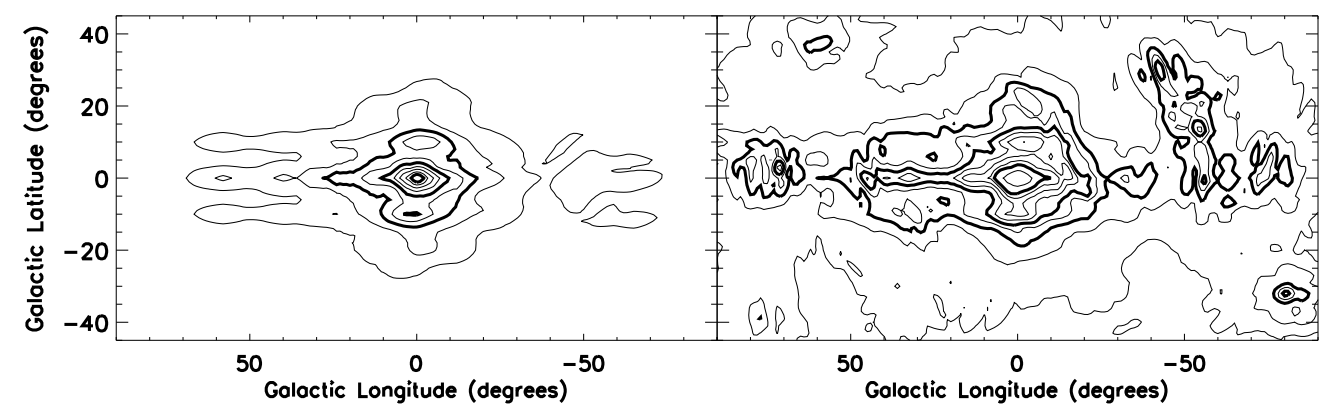

FIGURE 1. The exposure map of the expanded data-set compared with the earlier data-set of Purcell et al. (1997). The maps are in units of $10^{9} \mathrm{~cm}^{2} \mathrm{~s}$, with the contour levels $0.05,0.25,0.5$, $0.75,1,1.25,1.5,1.75,2,2.5$.

The combined OSSE/SMM data-sets are used rather than the OSSE-only dataset because the OSSE background-subtraction technique leads to differential rather than absolute fluxes. OSSE is insensitive to both isotropic emission and modest intensity gradients. The SMM fluxes are not absolute either, being insensitive to isotropic emission. Assuming isotropic emission to be zero, the SMM data contributes an overall normalization (Share et al. 1988). The SMM FoV is wide $\left(\sim 130^{\circ} \mathrm{FWHM}\right)$, but it does contribute limited spatial information as the response peak swept through the GC region along the ecliptic. One important difference between the PURC97 data-set and this one is the use of TGRS data. PURC97 used the TGRS data from Teegarden et al. (1996). That data has since been reanalyzed by Harris et al. (1998), with the resultant $511 \mathrm{keV}$ line flux reduced by almost 20\%. The Harris et al. (1998) data-set is used for the model-fitting studies shown in Table 1. SVD maps of the combined OSSE/SMM/TGRS data-set were not ready for these proceedings, but a RL map of the OSSE/SMM/TGRS data-sets (not shown) is in general agreement with the OSSE/SMM map.

\section{MAPPING POSITRON ANNIHILATION EMISSION}

Two techniques have been employed to map the OSSE data-set: minimizing $\chi^{2}$ with an adaptation of the Richardson-Lucy Algorithm (RL), and response matrix inversion using truncated Singular Valued Decomposition (SVD). SVD was described in PURC97, RL will be described in detail in an upcoming work. ${ }^{4}$ Differences between the two resulting maps suggest the level of the uncertainties involved.

The upper and middle panels of Figure 2 shows the SVD and RL $511 \mathrm{keV}$ maps of the combined OSSE and SMM data-sets. Both maps show three principle features, intense emission centered near the GC (hereafter called bulge emission), a fainter

4) In short, RL adds flux distributed according to the instrument response to the source/background regions to raise/lower individual flux values to match each observation. Through successive iterations, map structure develops and the overall $\chi^{2}$ lowers. 


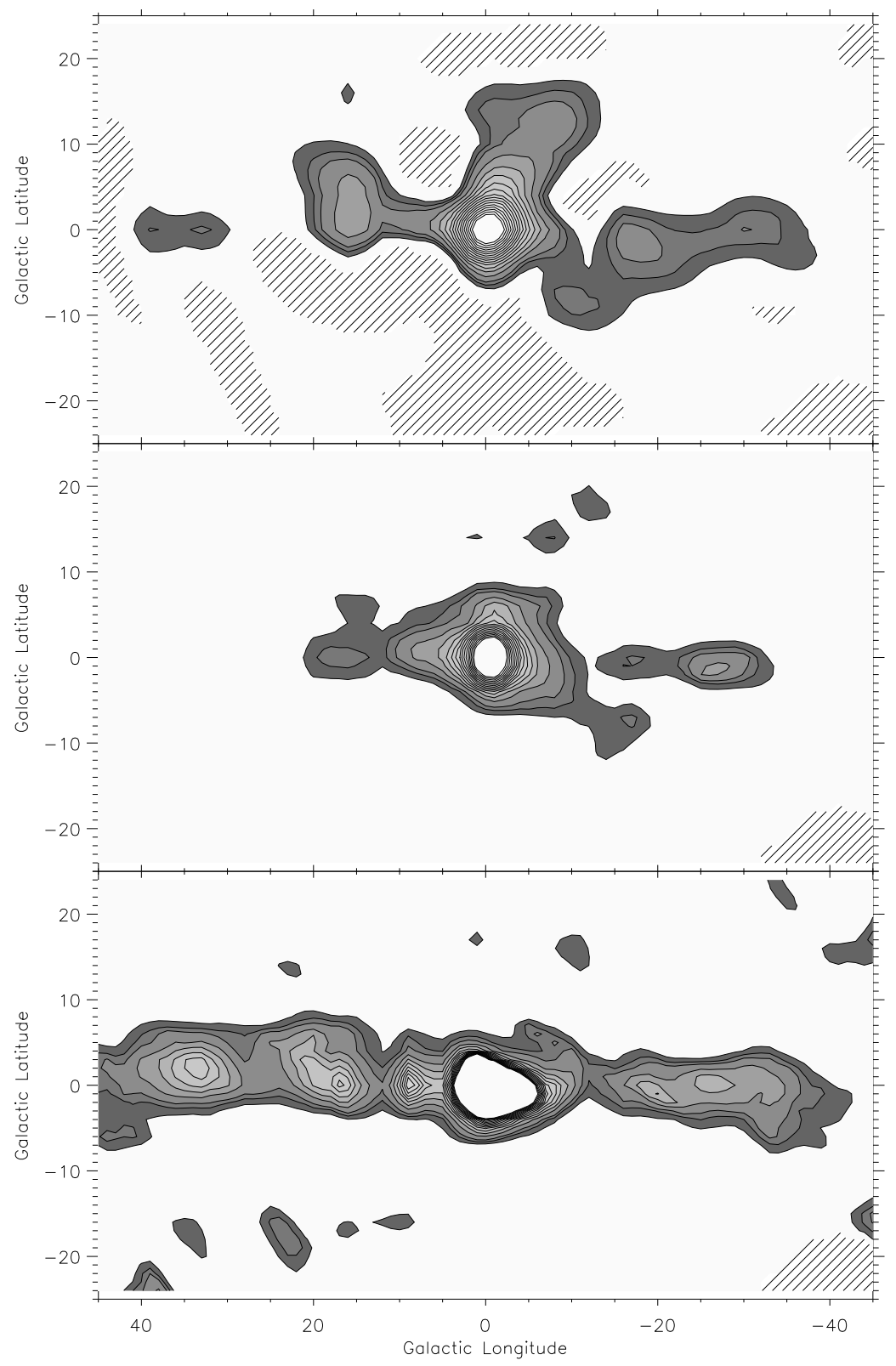

FIGURE 2. The SVD-511 map of the OSSE/SMM data-set is shown in figure 2a, the RL-511 map is shown in figure $2 \mathrm{~b}$. The RL-positronium continuum map of the OSSE-only data-set is shown in figure 2c. All contours are in increments of $2.3 \times 10^{-3}$ phot $\mathrm{cm}^{-2} \mathrm{~s}^{-1} \mathrm{ster}^{-1}$. For the RL maps, the hatched region is where the OSSE exposure does not meet a minimum threshold, for the SVD map the regions where unphysical negative fluxes occur is also hatched. 
planar emission (hereafter called disk emission), and emission from the negative longitude/positive latitude region (hereafter called a PLE). The PLE is more pronounced in the SVD map than in the RL map. The hatched region does not meet a minimum exposure threshold (or maps negative intensity). We emphasize the level to which the emission is concentrated in these three components. Although a far larger fraction of the inner radian is mapped than in PURC97, there is no indication of intense emission from any of these newly mapped regions. The apparent dominance of the bulge and disk emissions along with a contribution from the PLE drive the use of two and three component models when model-fitting.

Shown in the lower panel of Figure 2 is a RL map of the fitted positronium continuum fluxes of the OSSE data-set. In most astrophysical environments, more PCONT photons are produced in annihilation events than $511 \mathrm{keV}$ line photons. For a positronium fraction of 0.95 , the PCONT:511 ratio is $3.7: 1 .^{5}$ As a result, the PCONT map is more intense. As explained in the introduction, this map is preliminary as a number of potential biases have not yet been addressed. Nonetheless, the dominance of intense bulge and fainter disk components appears to agree with the $511 \mathrm{keV}$ maps. The principal difference is the lack of evidence of PLE emission, as will be discussed in the next section.

\section{LONGITUDE AND PLE CUTS}

A measure of the planar structure in the $511 \mathrm{keV}$ emission can be seen by taking a cut along the galactic plane of the RL and SVD maps. Cuts for the RL and SVD maps, as well as a model combining an $\mathrm{R}^{1 / 4}$ bulge and the DIRBE 100 disk are shown in the upper panel of Figure 3. Each is the sum of the $|b| \leq 2^{\circ}$ pixels. The SVD data is plotted with $1 \sigma$ error bars per degree. The SVD and RL 511 maps show rough agreement, except in the $+18^{\circ}$ to $+27^{\circ}$ region, where the peaks and valleys are exaggerated in the SVD map relative to the RL map. The model is more centrally peaked and the centroids of the maps are slightly offset towards negative longitude, but general agreement exists between the model and the maps. A systematic survey of the inner galactic plane scheduled for CGRO Cycle 9 will improve the sensitivity of the longitudinal cut.

The lower panel of Figure 3 shows a cut through the PLE at an angle of $60^{\circ}$ relative to the negative-longitude galactic plane (an angle suggested by the SVD map). As seen in the longitudinal cut, the $\mathrm{R}^{1 / 4}$ shape is more centrally peaked. In the anti-PLE direction, all three maps agree, all falling smoothly. Both the SVD511 and the RL-511 are brighter in the $+3^{\circ} \rightarrow+8^{\circ}$ region than the corresponding negative region. Beyond $+8^{\circ}$, the sensitivity becomes poor, and there is little significance to the differences between the SVD and RL maps. The PCONT map (not shown) does not show a corresponding enhancement, though interpretation of

5) OSSE has measured $\mathrm{f}_{P s}=0.97 \pm 0.03$ at the GC (Kinzer et al. 1996). This value is consistent with the TGRS measured value, $\mathrm{f}_{P s}=0.94 \pm 0.04$ (Harris et al. 1998). 

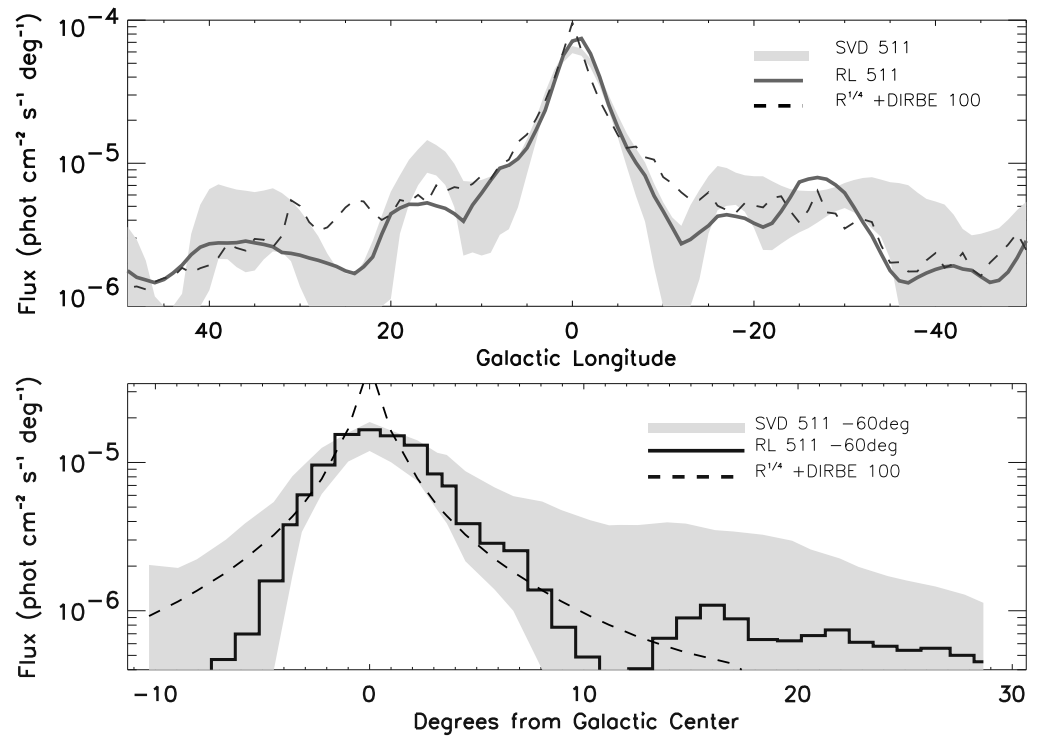

FIGURE 3. Figure 3a shows the integrated intensity from the $|b| \leq 2^{\circ}$ portion of the galactic plane. The SVD-511 cut is shown with $1 \sigma$ uncertainties, the RL-511 map and the $\mathrm{R}^{1 / 4}$ bulge and the DIRBE 100 disk model are shown without uncertainties. Figure $3 \mathrm{~b}$ shows a $1^{\circ}$ wide cut through the PLE region, determined to be best characterized as extending at a $60^{\circ}$ angle relative to the galactic plane. All maps were generated from the OSSE/SMM data-set.

this result as being due to annihilation physics, or alternatively being due to fitting systematics is not justified in this preliminary analysis. ${ }^{6}$

\section{MODEL-FITTING THE GALACTIC CENTER REGION}

The maps of $511 \mathrm{keV}$ line emission support the PURC97 representation of the galactic emission as being due to bulge, disk and PLE components. To quantify the contributions by the bulge and disk components, three bulge shapes have been combined with 28 disk shapes and compared with the OSSE, then the OSSE/SMM, then the OSSE/SMM/TGRS $511 \mathrm{keV}$ data-sets. The bulge shapes tested are; a GC point source, a Gaussian, and the projection of a truncated $\mathrm{R}^{1 / 4}$ function. Shown in the left panel of Figure 4 are the chi-squared values for best-fitting bulges of each shape paired with 28 disk models and fit to the OSSE data-set. The disks have

6) The "annihilation fountain" model (Dermer \& Skibo 1997) would have a low positronium fraction due to the high temperature, as direct annihilation with free electrons dominates over radiative recombination above $10^{6} \mathrm{~K}$ (Bussard, Ramaty \& Drachman 1979). However, the absence of broad emission in the TGRS spectra provides a constraint to this scenario (Harris et al. 1998). 


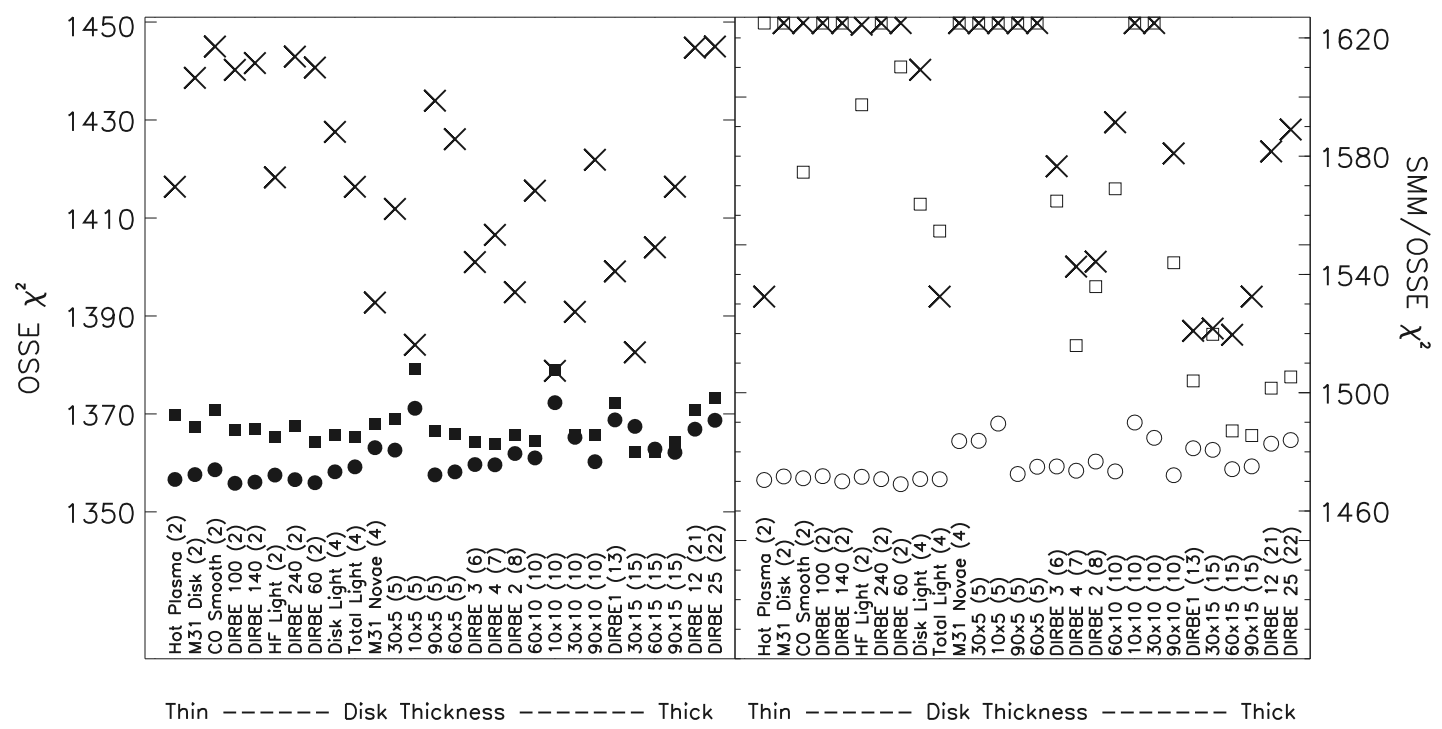

FIGURE 4. Results of bulge-disk fitting to OSSE-only (left panel) and OSSE/SMM (right panel) data-sets. The equivalent latitude FWHM of the disks are shown in parentheses. "90x10", etc. refers to the FWHM of disks with a Gaussian profile in width and thickness. The $\mathrm{R}^{1 / 4}$ bulges (filled and open circles) and Gaussian bulges (filled and open squares) fit both data-sets better than does the point source $(\mathrm{X})$. When fitting the OSSE data-set, thin disks are favored for the $\mathrm{R}^{1 / 4}$ shape, but disfavored for the Gaussian shape. The combined OSSE/SMM data-set strengthens these tendencies. Very large $\chi^{2}$ values for point source \& Gaussian fits were truncated to 1622 .

been ordered by the equivalent latitude FWHM of a Gaussian profile. ${ }^{7}$ In the fits, the FWHM of the Gaussian bulges have been permitted to vary (best-fit FWHM values range from $3.9^{\circ}$ to $\left.5.7^{\circ}\right)$. The $\mathrm{R}^{1 / 4}$ radial function has been truncated to a constant value inside of a radius $\left(\mathrm{R}_{\min }\right){ }^{8} \mathrm{R}_{\text {min }}$ has been constrained to be between $50 \mathrm{pc}$ and $700 \mathrm{pc}$. The radial distribution has then been projected onto the line of sight assuming the $\mathrm{GC}$ to be $8 \mathrm{kpc}$ distant. For all $\mathrm{R}_{\min }$, the $\mathrm{R}^{1 / 4}$ has extended wings relative to the Gaussian shape; for small $\mathrm{R}_{\text {min }}$, the $\mathrm{R}^{1 / 4}$ is also more centrally peaked. For every disk tested, the extended bulges are strongly favored over the GC point source. The $\mathrm{R}^{1 / 4}$ bulge shows a slight preference for thin disks, while the Gaussian bulge shows a stronger preference for thicker disks. This is interpreted as a hint of emission separated from both the galactic plane and the GC. For the $\mathrm{R}^{1 / 4}$ bulge shape, the extended bulge wings account for this emission. For the Gaussian, a thicker disk is required.

In the right panel of figure 4, the SMM data is combined with the OSSE data.

7) The map references are: DIRBE maps (Hauser et al. 1998), the CO map (Dame et al. 1987), the Hot Plasma (Koyama et al. 1989), the $\mathrm{R}^{1 / 4}$, HF Light \& Disk Light (Higdon \& Fowler 1987), the M31 maps (Ciardullo et al. 1987). "90x10", etc. refer to the FWHM of Gaussian disks.

8) The $\mathrm{R}^{1 / 4}$ function is $\rho(\mathrm{R} \leq 0.24)=\mathrm{A} \cdot \mathrm{R}^{-6 / 8} \cdot\left\{\exp \left(-\mathrm{B} \cdot \mathrm{R}^{1 / 4}\right)\right\}, \rho(\mathrm{R} \geq 0.24)=5 / 4 \cdot \mathrm{A} \cdot \mathrm{R}^{-7 / 8}$ $\left\{\exp \left(-\mathrm{B} \cdot \mathrm{R}^{1 / 4}\right)-\mathrm{C} \cdot \mathrm{R}^{-1 / 4}\right]$, where $\mathrm{R}=$ radial distance from $\mathrm{GC}$ in $\mathrm{kpc}$, and $\mathrm{A}, \mathrm{B}, \mathrm{C}$ are constants. 
TABLE 1. Fit parameters for better-fitting models to the OSSE-only, OSSE/SMM \& OSSE $/ \mathrm{SMM} /$ TGRS data-sets $(\mathrm{HP}=$ Hot Plasma, $\mathrm{DL}=$ Disk Light). Bulge emission is dominant for $\mathrm{R}^{1 / 4}$ pairs, while disk emission is dominant for Gaussian pairs. The Richardson-Lucy and SVD map parameters are shown for comparison.

\begin{tabular}{|c|c|c|c|c|c|c|c|c|c|c|c|c|}
\hline \multirow{2}{*}{$\frac{\text { Models }}{\mathbf{R}^{1 / 4}}$} & \multicolumn{4}{|c|}{ OSSE-only } & \multicolumn{4}{|c|}{ OSSE/SMM } & \multicolumn{4}{|c|}{ OSSE/SMM/TGRS } \\
\hline & $\mathrm{R}_{\min }$ & $\mathrm{B} / \mathrm{D}$ & $\mathrm{F}_{T}^{\mathrm{a}}$ & $\chi^{2} / \alpha$ & $\mathrm{R}_{\min }$ & $\mathrm{B} / \mathrm{D}$ & $\overline{\mathrm{F}_{T}{ }^{a}}$ & $\chi^{2} / \alpha$ & $\mathrm{R}_{\min }$ & $\mathrm{B} / \mathrm{D}$ & $\mathrm{F}_{T}^{a}$ & $\chi^{2} / \alpha$ \\
\hline $\mathrm{HP}$ & $100 \mathrm{pc}$ & 1.6 & 25.7 & 1.18 & $200 \mathrm{pc}$ & 1.7 & 28.3 & 1.20 & $200 \mathrm{pc}$ & 1.2 & 27.6 & 1.20 \\
\hline $\mathrm{CO}$ & $50 \mathrm{pc}$ & 2.6 & 30.0 & 1.18 & $50 \mathrm{pc}$ & 1.2 & 28.9 & 1.20 & $50 \mathrm{pc}$ & 3.3 & 31.3 & 1.20 \\
\hline DL & $50 \mathrm{pc}$ & 2.7 & 25.2 & 1.18 & $50 \mathrm{pc}$ & 1.5 & 29.0 & 1.20 & $50 \mathrm{pc}$ & 0.8 & 28.2 & 1.20 \\
\hline $90 \times 10$ & $50 \mathrm{pc}$ & 0.6 & 27.4 & 1.18 & $50 \mathrm{pc}$ & 0.9 & 27.9 & 1.20 & $50 \mathrm{pc}$ & 0.6 & 26.8 & 1.20 \\
\hline Gaus. & Fwhm & $\mathrm{B} / \mathrm{D}$ & $\overline{\mathrm{F}_{T}{ }^{a}}$ & $\overline{\chi^{2} / \alpha}$ & Fwhm & $\mathrm{B} / \mathrm{D}$ & $\overline{\mathrm{F}_{T}{ }^{a}}$ & $\chi^{2} / \alpha$ & Fwhm & $\mathrm{B} / \mathrm{D}$ & $\overline{\mathrm{F}_{T}{ }^{a}}$ & $\chi^{2} / \alpha$ \\
\hline $60 \times 15$ & $4.4^{\circ}$ & 0.2 & 20.9 & 1.19 & $4.3^{\circ}$ & 0.2 & 26.4 & 1.21 & $4.3^{\circ}$ & 0.2 & 26.4 & 1.21 \\
\hline $90 x 15$ & $5.1^{\circ}$ & 0.3 & 21.6 & 1.19 & $4.8^{\circ}$ & 0.2 & 29.6 & 1.32 & $5.7^{\circ}$ & 0.2 & 29.9 & 1.21 \\
\hline DRB12 & $5.5^{\circ}$ & 0.1 & 29.9 & 1.28 & $5.7^{\circ}$ & 0.2 & 31.3 & 1.33 & $5.7^{\circ}$ & 0.2 & 31.4 & 1.22 \\
\hline \multicolumn{2}{|c|}{ RL Map } & & 38.3 & 1.13 & & 0.63 & 37.0 & 1.14 & & & & \\
\hline \multicolumn{2}{|c|}{ SVD Map } & & 31.1 & 1.15 & & 0.49 & 25.8 & 1.18 & & & & \\
\hline
\end{tabular}

${ }^{\mathrm{a}}$ The total flux in units of $10^{-4}$ phot $\mathrm{cm}^{-2} \mathrm{~s}^{-1}$.

$\mathrm{b}$ The degrees of freedom have been set equal to those of the models.

The fits for the $\mathrm{R}^{1 / 4}$ solutions are similar to the OSSE-only plots. However, only the thicker disk-Gaussian bulge solutions are able to approximate the quality of the $\mathrm{R}^{1 / 4}$ fits. As seen in Table 1, the $\mathrm{B} / \mathrm{D}$ ratios are larger for $\mathrm{R}^{1 / 4}$ solutions than for Gaussian solutions. The total flux of the $\mathrm{R}^{1 / 4}$ solutions change little when SMM and then TGRS data is added, but for the Gaussian solutions the total flux can change considerably. For many Gaussian bulge-disk combinations, the OSSE data would not permit insertion of the SMM or TGRS-required flux without violating OSSE constraints, leading to poor fits. These solutions do not necessarily span the range of possible bulge-disk combinations, nor are they unique, but they do suggest ranges of plausible $\mathrm{B} / \mathrm{D}$ ratios and total fluxes. The uncertainties of the parameters are not shown in Table 1 due to concern about them being misinterpreted. The standard approach is to fix all but a single parameter, and calculate the degradation to the fit that results from varying that single parameter. The uncertainties that result do not account for the effect of varying the other parameters, nor does it account for other potential model shapes. In this paper, the uncertainties are evident in Table 1, but are not calculated.

The results shown in Table 1 have ignored the existence of a PLE and its potential influence upon the $\mathrm{B} / \mathrm{D}$ and $\mathrm{F}_{\text {Tot }}$ parameters. PURC97 quantified the PLE flux by two methods; (1) by subtracting the mirror region from the outputs of the mapping techniques, and (2) by fitting the data-set with three components (bulge, disk and PLE), all with Gaussian profiles. The two methods yield very different PLE flux results. The PURC97 maps suggest the PLE flux to be (1.3-1.5) x $10^{-4}$ phot $\mathrm{cm}^{-2}$ $\mathrm{s}^{-1}$. The 3-Gaussian fitting suggests (5.4-8.8) $\times 10^{-4}$ phot $\mathrm{cm}^{-2} \mathrm{~s}^{-1}$. The current maps suggest $(1.2 \pm 0.5) \times 10^{-4}$ phot $\mathrm{cm}^{-2} \mathrm{~s}^{-1}$, in agreement with the PURC97 
maps. Quantifying the PLE by mirror-region subtraction, the B/D ratios for the RL and SVD maps are 0.63 and 0.49 , as shown in Table 1 . The B/D values are intermediate to the $\mathrm{R}^{1 / 4}$ and Gaussian solutions, suggesting a general agreement between mapping and two component modeling.

The PURC97 3-Gaussian solution, when inserted with quoted parameters, is not an acceptable solution for the current OSSE/SMM/TGRS data-set $\left(\chi^{2} / \alpha=1.34\right)$, but the same method can be applied to examples of better-fitting models from Table 1. When a Gaussian representing the PLE is added to a $4.3^{\circ}$ Gaussian bulge $+60^{\circ} \times 15^{\circ}$ Gaussian disk, and the B/D re-optimized, the B/D rises from 0.163 to $0.169\left(\Delta \chi^{2}=-16.4\right)$. The PLE centroid for this Gaussian fit is determined to be $(l, b)=-2^{\circ},+8^{\circ}$, and the PLE flux is $1.1 \times 10^{-4}$ phot $\mathrm{cm}^{-2} \mathrm{~s}^{-1}$. When a PLE Gaussian is added to the $\mathrm{R}^{1 / 4}+\mathrm{CO}$ Smooth model, the B/D lowers slightly from 3.3 to $2.6\left(\Delta \chi^{2}=-12.3\right)$, and the parameters become: $(l, b)=-2^{\circ},+1^{\circ}$, PLE flux $=0.7 \times 10^{-4}$ phot $\mathrm{cm}^{-2} \mathrm{~s}^{-1}$. These values are given not to suggest a refinement of the PLE, but rather to demonstrate that the B/D depends more on the bulge shape than on the PLE (and vise versa). The current modeling of the PLE lowers the flux values to better agreement with the mapping fluxes, but the interpretation must be that the characteristics of any possible PLE are too poorly constrained and too model dependent to claim flux values and emission centroids (and the corresponding uncertainties).

\section{DISCUSSION}

OSSE investigations of galactic positron annihilation have recently been ex-

panded into three new areas; utilization of archival data near the GC, utilization of archival data away from the GC and mapping the positronium continuum emission. This expansion makes the results more dependent upon the spectral analysis, which is currently in its preliminary stage. The portion of this analysis least dependent upon these new complications are the $511 \mathrm{keV}$ line flux values. Although the spatial coverage of maps from these values has increased, characterization of the emission with three components (bulge, disk and PLE) remains adequate. An extended bulge emission has been shown to be favored over point-like emission from the GC for a collection of 28 disk models. Comparing two approximations of the bulge shape, Gaussian bulges require thick disks to fit the data-sets, while $\mathrm{R}^{1 / 4}$ bulges are less dependent upon the disk thickness, slightly favoring thin disks. The bulge shape must be better resolved with future measurements to improve the constraints upon the $\mathrm{B} / \mathrm{D}$ ratio (currently ranging from $0.2-3.3$ for the combinations tested). The total flux is better constrained, ranging between (20.9-31.4) $\times 10^{-4}$ phot $\mathrm{cm}^{-2}$ $\mathrm{s}^{-1}$. The effect of a potential PLE upon these parameters is shown to be less than the bulge-shape uncertainty when the PLE is approximated by a Gaussian.

Positron astronomy is 30 years old but remains in its infancy. Of current generation gamma-ray telescopes, OSSE is the best-suited to investigate the sky distribution of this emission. The time allotted to OSSE annihilation studies has increased 
in recent cycles of the CGRO mission. This time, combined with the expanded OSSE analysis, and the impending launch of the INTEGRAL telescopes should produce significant improvements of our understanding of the nature of galactic positron annihilation.

\section{REFERENCES}

1. Bussard, R.W., Ramaty, R. \& Drachman, R.J., ApJ 228, 928 (1979).

2. Cheng, L.-X., et al., ApJ 481, L43 (1997).

3. Ciardullo, R., et al., ApJ 318, 520 (1987).

4. Dame, T.M. et al., ApJ 322, 706 (1987).

5. Dermer, C.D. \& Skibo, J.G., ApJ 487, L57 (1997).

6. Harris, M.J., et al., ApJ 501, L55 (1998).

7. Hauser, M.G., et al., COBE Ref Pub No. 98-A Greenbelt, MD:NASA/GSPC, (1998).

8. Higdon, J.C. \& Fowler, W. A., ApJ 317, 710 (1987).

9. Kinzer, R.L., et al., A $\& A S$ 120, 317 (1996).

10. Milne, P.A., et al., Proceedings of the 3rd INTEGRAL Workshop in press, (1998).

11. Purcell, W.R., et al., ApJ 413, L85 (1993).

12. Purcell, W.R., et al., ApJ 491, 725 (1997).

13. Share, G.H., et al., ApJ 326, 717 (1988).

14. Share, G.H., et al., ApJ 358, L45 (1990).

15. Teegarden, B., et al., ApJ 463, L75 (1996). 